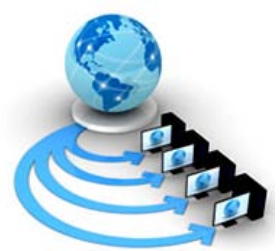

Volume 9, No. 2, March-April 2018

International Journal of Advanced Research in Computer Science

REVIEW ARTICLE

Available Online at www.ijarcs.info

\title{
A COMPREHENSIVE STUDY ON SMART CITY: CONCEPT AND LIMITING FACTORS
}

\author{
Rahul \\ Research Scholar \\ MDU Rohtak, India
}

\author{
Kamna Solanki \\ Assistant Professor \\ MDU Rohtak, India
}

\begin{abstract}
The notion of smart cities has become extremely popular over the recent years. The concept is being accepted and implemented globally not only by researchers but by industries too. A city can be assigned with the smart tag if it has certain features like well planned, provides efficient services to its residents, has intelligent infrastructure and these features in turn result in increase in quality of life.This paper is aimed at providing a review on in-depth insight into the conception of a Smart City.
\end{abstract}

Keywords: Smart City, Smart Healthcare, Smart Transport, Smart Parking.

\section{INTRODUCTION}

In current scenario, our cities are facing grim problems such as high carbon footprint, inefficient transportation system, ineffective healthcare and other public services. Most of these predicaments can be assigned to the higher rate of migration to the cities. A smart city can be an effective answer to these problems.

The concept of smart city is explained by many researchers, interest groups or stakeholderson the basis of their interpretation of the term "smart", and therefore there is no single globally accepted definition.The general impression is that a smart city is a city where technology\& innovation is used for betterment of the quality of life by optimizing the resources and by improving infrastructure and public services.

Definitions of Smart City: Washburn et al. define a smart city as "the use of smart computing technologies to make the critical infrastructure components and services of a city which include city administration, education, healthcare, public safety, real estate, transportation, and utilities - more intelligent, interconnected, and efficient"[1].

Caragliu et al. states "We believe a city to be smart when investments in human and social capital and traditional (transport) and modern (ICT) communication infrastructure fuel sustainable economic growth and a high quality of life, with a wise management of natural resources, through participatory governance”[2].

Aurigi also define smart city as a city having ICT (Information \& Communication Technology) as a vertebrafor all its operations[3].

Geller states "A city well performing in a forward-looking way in economy, people, governance, mobility, environment, and living, built on the smart combination of endowments and activities of self-decisive, independent and aware citizens”[4].

\section{SMART CITY MATURITY MODEL}

IDC Government Insights provides a smart city maturity model [5].

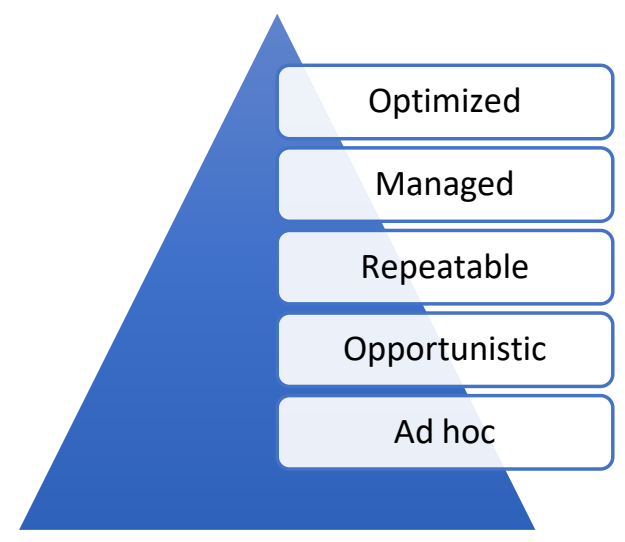

Figure 1. Smart City Maturity Model

It describes the stages of development of a smart city. A city can not be simply smart or dumb. This model states that a city can be in any of the stages i.e. Ad-hoc, opportunistic, repeatable, managed, optimized.

i) Ad-hoc: at this level government does not have any formal process to implement new concepts.

ii) Opportunistic:at this level experimentations are done with people by means of mobile apps and community networking.

iii) Repeatable: this level encourages recurring communications between citizens and governments.

iv) Managed: at this level innovation is organised.

v) Optimized: this is the highest maturity level a city can achieve, this refers to the state where the government has perfected innovation models.

A city can start from ad-hoc level and can gradually attain the status of optimized. 


\section{LITERATURE REVIEW}

According to Giffinger et al. smart cities must have these six characteristics[6]:

1. Smart Economy: This includes the factors that result in all around economic growth of the city. This specifically refers to improving innovation \& productivity and growth in field of Information \& Communication Technology.

2. Smart People:Smart people refers to the fact that citizens in a smart city not only should be having higher academic qualifications but they should be highly capable in interaction within the community and to the outer world.

3. Smart Governance: Smart Governance generally refers to e-governance, which aims at better association between administration and the citizens, also at delivering public services to its citizens efficiently.

4. Smart Mobility:It explains the need and solution for smart ways for deployment of transportation resources, better traffic management, and effective parking solutions.

5. Smart Environment: This pointsthe factors forreduction of pollution and providing better natural living conditions.

6. Smart Living: It describes the factors for achieving higher quality of life, such as safety, education and cultural facilities, social interaction events, healthcare services etc.

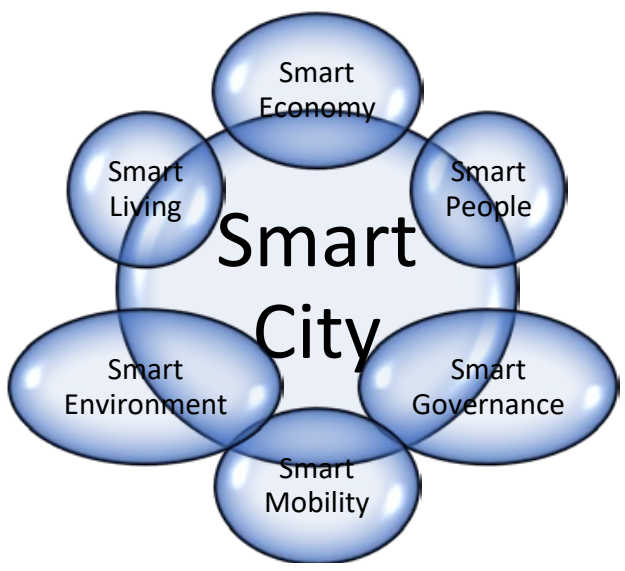

Figure 2. Characteristics of a Smart City

According to Ministry of Urban Development, Govt. of India, the core infrastructure in a smart city should include the elements given in figure 3 [7].

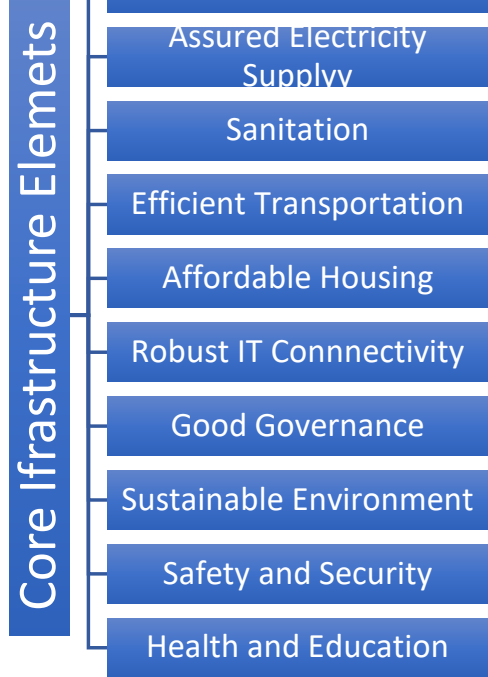

Figure 3. Core Infrastructure elements of a Smart City

These guidelines also provide smart solutions to achieve the vision of converting a city into smart city. For good governance, e-governance should be opted, so that all the public domain services are easily accessible to the citizens and transparency can be assured. For efficient waste management, the waste should be properly collected and recycled, organic waste should be converted to energy, fuel or compost. Waste water should be treated effectively. Water Supply management should be through smart meters with water quality monitoring and leakage identification and prevention. For efficient energy management, renewable sources of energy should be adopted, energy efficient architecture should be used for building construction. For efficient transportation, intelligent transportation management, smart parking and integrated multi-modal transport should be implemented.

Hall focuses that a smart city must aim at monitoring of its resources like various transportation modes, fluid supply system, buildings and its communications system using advanced sensors. As only then we can efficiently deploy its resources and is possible to cost effectively manage the city. Hall refers to the study on New York, USA[8].

The concept of smart cities cannot flourish without the help of industry giants. Some of the tech- industry titans have been working for realizing the smart cities into the real world. CISCO and IBM have been contributing in making the concept of smart city reality[9] [10].

IBM states that infrastructure and operations along with people provide the base for the concept of smart city. On the basis of these three factors all the services that make a city "smart", can be classified into three categories services concerning individuals, infrastructure related services and last but not the least planning and administrative services [11].

Casile depicts the characteristics of a smarter world as i)instrumented:as we can sense, measure and record everything ii) interconnected: as everything is connected and can communicate with each other (the concept of Internet of Things:IoT)iii) intelligent: as by analyzing 
quickly and accurately the data gathered, we can predict and prepare ourselves for forthcoming events [10].

Lobaccaro et al. explains the need for smart grids in smart cities. The paper compares various home energy management systems (HEMS) and concludes that by using HEMS, one can efficiently use electricity for reducing peak load, which in turn reduces the expenses on electricity bills and lowering ecosystem footprints [12].

$\mathrm{Ni}$ et al. focuses on smart healthcare in smart home environments. This paper describes the use of smart healthcare services for three purposes: i) health monitoring for a particular disease, ii) day-to-day reminders, predictions and monitoring iii) aberrant condition. recognizing aberrant condition [13].

Manoharan et al. describes the smart services of a smart city asi) smart energy metering: for efficient delivery and lowering energy consumption by analyzing the usage patterns, ii) smart fluid delivery system: for monitoring of water usage \& quality, efficient water distribution,iii) air quality monitoring system:for real time monitoring of air pollution levels, harmful gas detection, humidity \& temperature change detection and iv)condition monitoringof buildings and structures: for monitoring, detection and locating the damage on them [14].

\section{LIMITING FACTORS}

During practical implementation of smart cities there are a few limiting factors. These factors are given here:

1. Increasing Urban Population:Population growing at such an alarming rate and high migration rate to the cities poses a critical challenge to the realization of smart cities as with the increase in population the implementation \& integration of various services becomes more and more difficult.

2. Higher Cost of Implementation: At current stage the components required and the services necessary for smart cities are expensive. This results in higher cost of implementation.

3. Less $R \& D$ : This is a relatively new concept and there has been comparatively less Research in this field. Exhaustive research in any the field will also result in efficient implementation.

4. Security and Privacy Concerns: As the implementation of smart city relies on networks, it has all the flaws and limitations of networks. According to Cisco 50 billion devices will be connected. For better results, new networking technologies and better network protocols must be designed[15].

5. Traffic Congestion: Traffic congestion poses a grave concern for efficient transportation system. The transport system is highly overloaded and a significant amount of money is wasted due to congestion every year [16].

6. High Energy Consumption: Energy consumption in average home or office is relatively high, and can be reduced if smart solutions are implemented.

7. Dependency on Conventional Energy sources: We have been relying on conventional/ non-renewable sources for a very long time. For smart environment, we have to switch to other options very soon.

8. Healthcare Limitations:Healthcare is one of the most important and expensive sector. Healthcare services are either not accessible to a large portion of population or it is pricey in most of the countries. E-health can be a better solution in these conditions [17].

9. Large amount of data: While implementing smart cities, we have to deal with a large amount of data, and so new techniques have to be devised for its analysis and processing.

\section{CONCLUSION}

Smart cities propose a better living environment with a higher living standard. A city does not become "smart" only by technological advancements, but it is collective growth of infrastructure, the community and the citizens residing in the city.Any city can be transformed into a smart city by following the guidelines proposed by smart city maturity model. Furthermore, a smart city should opt smart solutions for healthcare, transportation and otherpublic services.

\section{REFERENCES}

[1] Washburn D., Sindhu U., Balaouras S., Dines R.A., Hayes N.M. and Nelson L.E., "Helping CIOs Understand 'Smart City' Initiatives: Defining the Smart City, its Drivers, and the Role of the CIO", Cambridge, MA: Forrester Research, Feb. 2010.

[2] Caragliu A., Del Bo C. and Nijkamp P.,"Smart cities in Europe”, Journal of Urban Technology, 2011.

[3] Aurigi A., "Making the Digital City: The Early Shaping of Urban Internet Space”, Farnborough: Ashgate, 2005.

[4] Geller, A. L., "Smart growth: a prescription for livable cities”, American Journal of Public Health, 93(9), 1410e1415, 2003.

[5] Clarke, R.Y., "Smart Cities and the Internet of Everything: The Foundation for Delivering NextGeneration Citizen Services”, IDC Government Insights, 2013.

[6] Giffinger, R., Fertner, C., Kramar, H., Meijers, E., "Smart cities: Ranking of European medium-sized cities”, Vienna University of Technology, 2007.

[7] Ministry of Urban Development Govt. of India, "Smart Cities: Mission Statement \& Guidelines”, June 2015.

[8] Robert E. Hall, "The Vision of a Smart City" 2nd International Life Extension Technology Workshop, 2000.

[9] Falconer G., Mitchel S., "Smart City Framework: A systematic Process for enabling smart + connected communities”, Cisco Internet Business Solutions Group, September, 2012.

[10] Casile O., "IBM, for a Smarter Planet and Smarter Cities”, EMEA IBM Innovation Center La Gaude, France, July 6-8, 2011.

[11] Sderstrm O., "Paascies as corporate storytelling, City: analysis of urban trends, culture, theory, policy, action”, 18:3, 307-320, 2014.

[12] Lobaccaro G., Carlucci S., Löfström E., “A Review of Systems and Technologies for Smart Homes and Smart Grids”, Energies 2016, 9, 348; d Grids, Energies 2016, 9, 348; doi:10.3390/en9050348

[13] Ni Q., Hernando A.B.G., Cruz I.P., “The Elderly’s Independent Living in Smart Homes: A Characterization of Activities and Sensing Infrastructure Survey to 
Facilitate Services Development”, Sensors 201s150511312.

[14] Manoharan A.M., R. V., "Smart City: Issues and Research Challenges in Implementation", International Conference on Smart Grid and Smart Cities, IEEE, 2017.

[15] "Building the Hyperconnected Society IoT Research and Innovation Value Chains Ecosystems and Markets", River publishers Series in Communication, 2015.

[16] "Clean transport, European Commission, DirectorateGeneral for Mobility and Transport”. http://ec.europa.eu/transport/themes/urban/urban mobility/index en.htm, 2015.

[17] Martin R. Cowie, JeroenBax, Nico Bruining, John G. F. Cleland, Friedrich Koehler, Marek Malik, Fausto Pinto, Enno van der Velde, and PanosVardas, "e-Health: a position statement of the European Society of Cardiology”, European Heart Journal, 2015. 